\title{
HOMOGENEOUS QUATERNIONIC KÄHLER STRUCTURES ON ALEKSEEVSKIAN $\mathcal{W}$-SPACES
}

\author{
WAFAA BATAT, PEDRO M. GADEA, AND JAIME MUÑOZ MASQUÉ
}

Abstract. The homogeneous quaternionic Kähler structures on the Alekseevskian $\mathcal{W}$-spaces with their natural quaternionic structures, each of these spaces described as a solvable Lie group, and the type of such structures in Fino's classification, are found.

\section{INTRODUCTION}

Quaternion-Kähler manifolds have attired much attention from the classical papers by Wolf $[\mathrm{W}]$, Ishihara $[\mathrm{I}]$ and others to now, see for instance $[\mathrm{J}]$ and $[\mathrm{V}]$, among many papers.

A quaternion-Kähler manifold is said to be negative if it is complete and has negative scalar curvature. Homogeneous quaternion-Kähler spaces admitting a simply transitive completely solvable Lie group of isometries were classified by Alekseevsky [A] (see also de Wit and van Proeyen [WP] and Cortés [Co]). No more homogeneous negative quaternion-Kähler spaces are known. Alekseevsky conjectured in [A, p. 300] that the only homogeneous negative quaternion-Kähler manifolds are Alekseevskian spaces.

Homogeneous quaternionic Kähler structures, i.e., the $\operatorname{Sp}(n) \operatorname{Sp}(1)$ case of Tricerri and Vanhecke [TV] homogeneous Riemannian structures, have been studied in [BGO1, BGO2, CGO1, CGO2, CGS, F]. Fino gave in [F, Lem. 5.1] a representation-theoretical classification of such structures, with five basic geometric types $\mathcal{Q} \mathcal{K}_{1}, \ldots, \mathcal{Q} \mathcal{K}_{5}$. (We denote the type $\mathcal{Q} \mathcal{K}_{i} \oplus \mathcal{Q} \mathcal{K}_{j}$ by $\mathcal{Q} \mathcal{K}_{i j}$, and so on.) A classification by real tensors was given in [CGS, Th. 1.1], and was also proved that a connected, simply-connected and complete homogeneous quaternion-Kähler manifold of dim $\geq 8$, admitting a nonvanishing structure in $\mathcal{Q} \mathcal{K}_{123}$ with nonzero projection to $\mathcal{Q} \mathcal{K}_{3}$, is isometric to the quaternionic hyperbolic space $\mathbb{H H}(n)$. Furthermore, a structure of type $\mathcal{Q} \mathcal{K}_{134}$ on $\mathbb{H} H(n)$, corresponding to its description as a solvable Lie group, has been given in [CGS, Prop. 5.3]. Then, in [CGO1, Th. 3.4] and [CGO2, Th. 5] it has been shown that the quaternion-Kähler symmetric spaces of

2010 Mathematics Subject Classification. Primary 53C26; Secondary 53C30.

Key words and phrases. Alekseevskian spaces, Quaternion-Kähler manifolds, Homogeneous quaternionic Kähler structures. 
dimension 8 or 12 furnish proper realisations of the types $\mathcal{Q} \mathcal{K}_{134}, \mathcal{Q} \mathcal{K}_{135}$, $\mathcal{Q} \mathcal{K}_{1345}, \mathcal{Q} \mathcal{K}_{12345}$. Fino's classification has been extended to any signature of the metric in [BGO1, Th. 4.4], and the structures on rank-three Alekseevskian spaces, $\mathcal{T}(p), p \geq 0$, endowed with their natural structure as solvable Lie groups, have been found in [BGO2, Th. 3.1].

Negative quaternion-Kähler spaces appear in $N=2$ supergravity. If gravity is considered as a dynamical field, the holonomy group of the manifold is a subgroup of $\operatorname{Sp}(n) \operatorname{Sp}(1)$ and $M$ is a negative quaternion-Kähler manifold (Bagger and Witten $[\mathrm{BW}]$ ). Cecotti [Ce] proved that Alekseevskian spaces naturally appear in the context of the $c$-map and that nonsymmetric ones are related to Vinberg $T$-algebras as symmetric ones are related to Jordan algebras. De Wit and van Proeyen [WP] completed Alekseevsky's classification, by using supergravity considerations. That Alekseevskian spaces do appear in three series, $\mathcal{T}$-, $\mathcal{W}$-, $\mathcal{V}$-spaces, was proved by Cortés [Co, Th. II.28] with geometric arguments.

Our aim is to give the expression of the homogeneous quaternionic Kähler structures carried by the rank-four Alekseevskian spaces $\mathcal{W}(p, q)$, each of them described as a solvable Lie group, and then their type in Fino's classification. To this end, we make calculations which are crucially based on the explicit description of the spaces $\mathcal{W}(p, q)$ as completely solvable Lie groups with a left-invariant quaternionic Kähler structure, given by Cortés in [Co].

After some preliminaries in $\S 2$, we obtain in $\S 3$ Theorem 3.1, giving the homogeneous quaternionic Kähler structure corresponding to the description of each space $\mathcal{W}(p, q)$ as a solvable Lie group. We give in $\S 4$ Theorem 4.1 , with the type of such structure, proving that it has nonzero components in each basic Fino's type.

\section{Preliminaries}

Ambrose and Singer [AS] proved that a connected, simply-connected and complete Riemannian manifold $(M, g)$ is Riemannian homogeneous if and only if it admits a homogeneous Riemannian structure, i.e., a $(1,2)$ tensor

field $S$ satisfying $\widetilde{\nabla} g=0, \widetilde{\nabla} R=0, \widetilde{\nabla} S=0$, where $\widetilde{\nabla}=\nabla-S, \nabla$ denotes the Levi-Civita connection and $R$ the curvature tensor of $\nabla$. We write as usual $S_{X Y Z}=g\left(S_{X} Y, Z\right)$. Then, from $\nabla g=0$ it follows that the condition $\widetilde{\nabla} g=0$ is equivalent to $S_{X Z Y}=-S_{X Y Z}$.

Let $\left(M, g, v^{3}\right)$ be an almost quaternion-Hermitian manifold. Let $J_{1}, J_{2}, J_{3}$ be a standard local basis of $v^{3}$ and let $\omega_{a}(X, Y)=g\left(J_{a} X, Y\right), a=1,2,3$. The differential 4-form $\Omega=\sum_{a=1}^{3} \omega_{a} \wedge \omega_{a}$ is known to be globally defined. 
The manifold is said to be quaternion-Kähler if one has locally (cf. Ishihara [I]) that

$$
\nabla_{X} J_{1}=\tau^{3}(X) J_{2}-\tau^{2}(X) J_{3}, \quad \text { etc. }
$$

for certain differential 1-forms $\tau^{1}, \tau^{2}, \tau^{3}$ ('etc.' denoting the equations obtained by cyclically permuting $1,2,3)$; or, equivalently, if $\nabla \Omega=0$.

We shall consider negative quaternion-Kähler manifolds of $\operatorname{dim} \geq 8$. A quaternion-Kähler manifold $\left(M, g, v^{3}\right)$ of $\operatorname{dim} \geq 8$ is said to be a homogeneous quaternion-Kähler manifold if ([AC, p. 218], cf. [CGS, Rem. 2.2]) it admits a transitive group of isometries. As a Corollary to Kiričenco's Theorem $[\mathrm{K}]$, a connected, simply-connected and complete quaternion-Kähler manifold $\left(M, g, v^{3}\right)$ is homogeneous if and only if there exists a tensor field $S$ of type $(1,2)$ on $M$ satisfying

$$
\widetilde{\nabla} g=0, \quad \widetilde{\nabla} R=0, \quad \widetilde{\nabla} S=0, \quad \widetilde{\nabla} \Omega=0,
$$

where $\widetilde{\nabla}=\nabla-S$. Such a tensor $S$ is called a homogeneous quaternionic Kähler structure on $M$. The equation $\widetilde{\nabla} \Omega=0$ is equivalent to conditions similar to (2.1).

Fino [F, Lem. 5.1] gave a representation-theoretical classification of homogeneous quaternionic Kähler structures, having five basic geometric types, which we denote by $\mathcal{Q} \mathcal{K}_{1}, \ldots, \mathcal{Q} \mathcal{K}_{5}$.

Let $(V,\langle\rangle, q$,$) be a quaternion-Hermitian vector space, i.e., a 4 n$-dimensional real vector space endowed with an inner product $\langle$,$\rangle and a quater-$ nionic structure q generated by suitable operators $J_{1}, J_{2}, J_{3}$. Consider the space of tensors $\mathcal{T}(V)=\left\{S \in \otimes^{3} V^{*}: S_{X Y Z}=-S_{X Z Y}\right\}$ and its vector subspace

$$
\begin{aligned}
\mathcal{Q K}(V)= & \left\{S \in \otimes^{3} V^{*}: S_{X Y Z}=-S_{X Z Y}, \exists \theta^{a} \in V^{*} \text { such that } S\right. \text { satisfies } \\
& \left.S_{X J_{1} Y J_{1} Z}-S_{X Y Z}=\theta^{3}(X) g\left(J_{2} Y, J_{1} Z\right)-\theta^{2}(X) g\left(J_{3} Y, J_{1} Z\right), \text { etc. }\right\}
\end{aligned}
$$

Any homogeneous Riemannian structure on $M$ belongs to $\mathcal{T}\left(T_{p} M\right)$ pointwise, but homogeneous quaternionic Kähler structures are pointwise in the space $\mathcal{Q K}\left(T_{p} M\right)$.

Consider the subspaces $\check{\mathcal{V}}$ and $\widehat{\mathcal{V}}$ of $\mathcal{Q} \mathcal{K}(V)$ consisting of elements $\Theta$ and $\mathcal{T}$, respectively, such that $\Theta_{X Y Z}=\sum_{a=1}^{3} \alpha^{a}(X)\left\langle J_{a} Y, Z\right\rangle, \alpha^{a} \in V^{*}$, and $\mathcal{T}_{X J_{a} Y J_{a} Z}=\mathcal{T}_{X Y Z}, a=1,2,3$. Then one has $\mathcal{Q K}(V)=\check{\mathcal{V}} \oplus \widehat{\mathcal{V}}$, and each element $S \in \mathcal{Q K}(V)$ decomposes as $S_{X Y Z}=\Theta_{X Y Z}+\mathcal{T}_{X Y Z}$, where

$$
\Theta_{X Y Z}=\frac{1}{2} \sum_{a=1}^{3} \alpha^{a}(X)\left\langle J_{a} Y, Z\right\rangle
$$


The classification by real tensors is ([CGS, Th. 3.15]) as follows: If $n \geq 2$, the space $\mathcal{Q K}(V)$ decomposes into the direct sum of the following $\operatorname{Sp}(n) \operatorname{Sp}(1)$ invariant and irreducible subspaces:

$$
\begin{aligned}
& \mathcal{Q} \mathcal{K}_{1}=\left\{\Theta \in \check{\mathcal{V}}: \Theta_{X Y Z}=\sum_{a=1}^{3} \alpha\left(J_{a} X\right)\left\langle J_{a} Y, Z\right\rangle, \alpha \in V^{*}\right\}, \\
& \mathcal{Q} \mathcal{K}_{2}=\left\{\Theta \in \check{\mathcal{V}}: \Theta_{X Y Z}=\sum_{a=1}^{3} \alpha^{a}(X)\left\langle J_{a} Y, Z\right\rangle, \sum_{a=1}^{3} \alpha^{a} \circ J_{a}=0, \alpha^{a} \in V^{*}\right\}, \\
& \mathcal{Q} \mathcal{K}_{3}=\left\{\mathcal{T} \in \widehat{\mathcal{V}}: \mathcal{T}_{X Y Z}=\langle X, Y\rangle \beta(Z)-\langle X, Z\rangle \beta(Y)\right.(2.4) \quad \\
&\left.\quad+\sum_{a=1}^{3}\left(\left\langle X, J_{a} Y\right\rangle \beta\left(J_{a} Z\right)-\left\langle X, J_{a} Z\right\rangle \beta\left(J_{a} Y\right)\right), \beta \in V^{*}\right\}, \\
& \mathcal{Q} \mathcal{K}_{4}=\left\{\mathcal{T} \in \widehat{\mathcal{V}}: \mathcal{T}_{X Y Z}=\frac{1}{6}\left(\underset{X Y Z}{\mathfrak{S}} \mathcal{T}_{X Y Z}+\underset{X J_{a} Y J_{a} Z}{\mathfrak{S}} \sum_{a} \mathcal{T}_{X J_{a} Y J_{a} Z}\right), c_{12}(\mathcal{T})=0\right\}, \\
& \mathcal{Q} \mathcal{K}_{5}=\left\{\mathcal{T} \in \widehat{\mathcal{V}}: \underset{X Y Z}{\mathfrak{S}} \mathcal{T}_{X Y Z}=0\right\},
\end{aligned}
$$

where $c_{12}(\mathcal{T})(Z)=\sum_{i=1}^{4 n} \mathcal{T}_{e_{i} e_{i} Z}$, for any local orthonormal basis $\left\{e_{i}\right\}$ of $V$.

We now recall some definitions and results by Alekseevsky [A] (cf. [AC], [Co]). A quaternion-Kähler manifold of nonzero scalar curvature is said to be an Alekseevskian space if it admits a simply transitive, completely solvable Lie group of isometries. An Alekseevskian space is simply-connected and it can be regarded as a completely solvable Lie group with a left-invariant metric. The corresponding metric Lie algebra with the quaternionic structure inherited from that of the manifold is a quaternion-Hermitian vector space $(\mathfrak{s},\langle\rangle, q$,$) , which is called a quaternionic or Alekseevskian Lie algebra.$ A metric Lie algebra $\mathfrak{f}$ with an orthonormal basis $\{G, H\}$ and a complex structure $J$ is said to be a key algebra with root $\mu$, if $G=J H,[H, G]=\mu G$, $\mu>0$. A metric Lie algebra $\mathfrak{f}+\mathfrak{x}$ with a complex structure $J$ is said to be an elementary Kählerian Lie algebra with root $\mu$ if $\mathfrak{f}=\operatorname{Span}\{G, H\}$ is a key subalgebra with root $\mu$ and $\left.\operatorname{ad}_{H}\right|_{\mathfrak{x}}=\frac{1}{2} \mu I,\left.\operatorname{ad}_{G}\right|_{\mathfrak{x}}=0,[X, Y]=\mu\langle J X, Y\rangle G$, $X, Y \in \mathfrak{x}$. A representation $U \mapsto T_{U}$ of a Lie algebra $\mathfrak{u}$ with a complex structure $J$ on a Euclidean space $(\mathfrak{x},\langle\rangle$,$) with a complex structure J_{1}$ is said to be symplectic if it satisfies two conditions given in [A, Def. 6.3]. If $T_{\mathfrak{u}} \mathfrak{x}=\mathfrak{x}$, $T$ is called nondegenerate. If $T$ is a nondegenerate symplectic representation of a key algebra $\mathfrak{f}=\operatorname{Span}\{G, H\}$ with root $\mu$ on a space $\left(\mathfrak{x},\langle\rangle,, J_{1}\right)$, then $\mathfrak{x}$ admits a weight decomposition $\mathfrak{x}=\mathfrak{x}_{+}+\mathfrak{x}_{-}$such that

$$
\mathfrak{x}_{-}=J_{1} \mathfrak{x}_{+},\left.\quad T_{G}\right|_{\mathfrak{x}_{+}}=0,\left.\quad T_{G}\right|_{\mathfrak{x}_{-}}=-\mu J_{1},\left.\quad T_{H}\right|_{\mathfrak{x}_{ \pm}}= \pm \frac{1}{2} \mu I .
$$

Any Alekseevskian algebra $(\mathfrak{s},\langle\rangle,, \mathrm{q})$, with $\mathrm{q}=\operatorname{Span}\left\{J_{a}: a=1,2,3\right\}$, contains a unique (up to scaling) one-dimensional quaternionic subalgebra $\mathfrak{s}^{\prime}$ (i.e., a subalgebra $\mathfrak{s}^{\prime}$ such that $q \mathfrak{s}^{\prime} \subset \mathfrak{s}^{\prime}$ ), corresponding either to the complex hyperbolic plane $\mathbb{C H}(2)$ or to the quaternionic hyperbolic line $\mathbb{H} H(1)$. In the 
former case it is of the form $\mathfrak{s}=\mathfrak{u}+J_{2} \mathfrak{u}$ (orthogonal sum), and ( $\left.\mathfrak{u}, J_{1 \mid \mathfrak{u}}\right)$ is the so-called principal Kählerian subalgebra of $\mathfrak{s}$. The Lie algebra $\mathfrak{u}$ contains a key subalgebra $\mathfrak{f}_{0}=\operatorname{Span}\left\{G_{0}, H_{0}\right\}$ with root 1 such that $\mathfrak{f}_{0}+J_{2} \mathfrak{f}_{0}$ is the canonical one-dimensional quaternionic subalgebra of $\mathfrak{s}$, and the adjoint representation of $\mathfrak{s}$ induces a representation of $\mathfrak{u}$ on $\mathfrak{u}^{\perp}=J_{2} \mathfrak{u}$. A Kählerian Lie algebra $(\mathfrak{u}, J)$, that is, a metric Lie algebra which corresponds to a Kählerian homogeneous space, is said to be admissible if $\mathfrak{u}=\mathfrak{f}_{0}+\mathfrak{u}_{0}$ is a direct orthogonal sum of a key algebra $\mathfrak{f}_{0}=\operatorname{Span}\left\{G_{0}, H_{0}\right\}$ with root 1 and a completely solvable Kählerian Lie algebra $\mathfrak{u}_{0}$. Denote $\widetilde{U}=\varphi(U)$ for each $U \in \mathfrak{u}$, and by $J_{1}$ and $\hat{J}$ the complex structures on $\tilde{\mathfrak{u}}$ given by

$$
J_{1}=-\varphi J \varphi^{-1}, \quad \hat{J}\left|\widetilde{\mathfrak{f}}_{0}=-J_{1} \widetilde{\mathfrak{f}}_{0}, \quad \hat{J}\right| \widetilde{\mathfrak{u}}_{0}=J_{1} \mid \widetilde{\mathfrak{u}}_{0} .
$$

Then a representation $U \mapsto T_{U}$ of such a Lie algebra $\mathfrak{u}$ on a Euclidean space $\tilde{\mathfrak{u}}$ together with a vector space isometry $\varphi: \mathfrak{u} \rightarrow \tilde{\mathfrak{u}}$ is said to be a $Q$-representation if it satisfies the eight conditions (Q1-8) given in [A, Lem. 5.5 and Def. 5.3] (cf. also Cortés [Co, Def. 1.8]).

If $\mathfrak{s}$ is an Alekseevskian Lie algebra with principal Kählerian subalgebra $(\mathfrak{u}, J)$, then the representation of $\mathfrak{u}$ on $J_{2} \mathfrak{u}$ induced by the adjoint representation of $\mathfrak{s}$ is a Q-representation with $\varphi=\left.J_{2}\right|_{\mathfrak{u}}: \mathfrak{u} \rightarrow \mathfrak{u}^{\perp}$. Conversely, let $(T, \varphi)$ be a Q-representation of an admissible Kählerian Lie algebra $(\mathfrak{u}, J)$ on the Euclidean vector space $\tilde{\mathfrak{u}}=\varphi(\mathfrak{u})=\tilde{\mathfrak{f}}_{0}+\widetilde{\mathfrak{u}}_{0}$. Then a quaternionic structure $\mathrm{q}=\operatorname{Span}\left\{J_{a}: a=1,2,3\right\}$ on the Euclidean vector space $\mathfrak{s}=\mathfrak{u}+\tilde{\mathfrak{u}}$ (orthogonal sum) is defined by

$$
\left.J_{1}\right|_{\mathfrak{u}}=J,\left.\quad J_{1}\right|_{\mathfrak{u}}=-\varphi J \varphi^{-1},\left.\quad J_{2}\right|_{\mathfrak{u}}=\varphi,\left.\quad J_{2}\right|_{\tilde{\mathfrak{u}}}=-\varphi^{-1}, \quad J_{3}=J_{1} J_{2} .
$$

Let $\hat{J}$ be the complex structure on $\tilde{\mathfrak{u}}$ defined as in (2.6), and let $\hat{\omega}$ denote the Kähler form on $\tilde{\mathfrak{u}}$ given by $\hat{\omega}(\widetilde{U}, \widetilde{V})=\langle\hat{J} \widetilde{U}, \widetilde{V}\rangle$. Then the following conditions define the structure of Lie algebra of $\mathfrak{s}$ :

$$
\mathfrak{u} \text { is a subalgebra of } \mathfrak{s},\left.\quad \operatorname{ad}_{U}\right|_{\tilde{\mathfrak{u}}}=T_{U}, \quad[\widetilde{U}, \widetilde{V}]=\hat{\omega}(\widetilde{U}, \widetilde{V}) G_{0},
$$

for all $U, V \in \mathfrak{u}$.

The rank of a solvable Lie algebra $\mathfrak{s}$ is the dimension of a Cartan subalgebra of $\mathfrak{s}$. The rank of an Alekseevskian space $\mathcal{S}$ is the rank of its Alekseevskian Lie algebra $\mathfrak{s}$, which is proved to be at most 4 . An admissible Kählerian Lie algebra $\mathfrak{u}=\mathfrak{f}_{0}+\mathfrak{u}_{0}$ which admits a Q-representation decomposes as a semidirect sum of elementary Kählerian Lie algebras, with $\mathfrak{u}_{0}=\sum_{i \geq 1}\left(\mathfrak{f}_{i}+\mathfrak{x}_{i}\right)$, that is, $\left[\mathfrak{f}_{i}+\mathfrak{x}_{i}, \mathfrak{f}_{j}+\mathfrak{x}_{j}\right] \subset \mathfrak{f}_{j}+\mathfrak{f}_{j}, i \geq j$, with symplectic representation $\operatorname{ad}_{\mathfrak{f}_{i}} \mid \mathfrak{x}_{j}$ for $i>j$ and commuting key algebras, $\left[\mathfrak{f}_{i}, \mathfrak{f}_{j}\right]=0$, for $i \neq j$ (see [Co, p. 134]). The rank of $\mathfrak{u}=\mathfrak{f}_{0}+\sum_{i \geq 1}\left(\mathfrak{f}_{i}+\mathfrak{x}_{i}\right)$ coincides with the 
number of key algebras of $\mathfrak{u}$. There are three types of admissible Kählerian Lie algebras, the first type corresponding to the case with smallest root 1 .

\section{Homogeneous quaternionic Kähler structures on $\mathcal{W}(p, q)$}

Now we focus on the rank-four $\mathcal{W}$-case and we shall make calculations essentially based on the explicit description, found by Cortés [Co], of the spaces $\mathcal{W}(p, q), 0 \leq p \leq q$, as completely solvable Lie groups with a leftinvariant quaternionic Kähler structure.

We recall that given Euclidean spaces $x, y, z$, a bilinear map $\psi: x \times z \rightarrow y$ is said to be isometric if $\langle\psi(X, Z), \psi(X, Z)\rangle=\langle X, X\rangle\langle Z, Z\rangle, X \in x, Z \in z$. Let $\mathfrak{x}_{-}, \mathfrak{z}_{-}, \mathfrak{y}_{-}$be Euclidean vector spaces. Every isometric map $\psi: \mathfrak{x}_{-} \times \mathfrak{z}_{-} \rightarrow$ $\mathfrak{y}_{-}$defines a Kählerian Lie algebra $\mathfrak{u}(\psi)=\left(\mathfrak{f}_{0}+\mathfrak{u}_{0}, J\right)$ of type 1 and rank 4 by means of a recipe given in [A, Prop. 9.3]. According to [A, Props. 9.29.4], there are two possibilities for Kählerian Lie algebras $\mathfrak{u}=\mathfrak{u}(\psi)$ of type 1 and rank $>2$ which admit a Q-representation. These two possibilities originate the series of Alekseevskian $\mathcal{W}$ - and $\mathcal{V}$-spaces, respectively. The $\mathcal{W}$-spaces correspond to the case $\mathfrak{x}_{-}=0$ (hence $\psi=0$ ), and $\mathfrak{u}=\mathfrak{u}(p, q) \cong$ $\mathfrak{u}(q, p)$ is completely determined by the parameters $p=\operatorname{dim} \mathfrak{y}_{-} \geq 0$ and $q=\operatorname{dim} \mathfrak{z}_{-} \geq 0$. Any such Lie algebra $\mathfrak{u}$ has a unique Q-representation $T$ and the corresponding Alekseevskian spaces are denoted by $\mathcal{W}(p, q)$. In this case the set of rules of the aforementioned recipe reduces to:

1. The space $\mathfrak{u}_{0}$ is a semidirect sum $\mathfrak{u}_{0}=\left(\mathfrak{f}_{1}+\mathfrak{x}_{1}\right)+\mathfrak{f}_{2}+\mathfrak{f}_{3}$ of elementary Kählerian key algebras with commuting Lie algebras with root 1 .

2. The space $\mathfrak{x}_{1}$ admits a $J$-invariant decomposition $\mathfrak{x}_{1}=\mathfrak{y}+\mathfrak{z}$ such that $\left.\operatorname{ad}_{\mathfrak{f}_{3}}\right|_{\mathfrak{y}}$ and $\left.\operatorname{ad}_{\mathfrak{f}_{2}}\right|_{\mathfrak{z}}$ are nondegenerate symplectic representations with weight decompositions $\mathfrak{y}=\mathfrak{y}_{+}+\mathfrak{y}_{-}$and $\mathfrak{z}=\mathfrak{z}_{+}+\mathfrak{z}_{-}$, where $\mathfrak{y}_{+}=J \mathfrak{y}_{-}$and $\mathfrak{z}_{+}=J \mathfrak{z}_{-}$. Furthermore, $\left[\mathfrak{f}_{2}, \mathfrak{y}\right]=\left[\mathfrak{f}_{3}, \mathfrak{z}\right]=[\mathfrak{y}, \mathfrak{z}]=0$.

Let $\left\{Y_{j+}\right\}, j=1, \ldots, p$, and $\left\{Z_{k+}\right\}, k=1, \ldots, q$, be orthonormal bases of $\mathfrak{y}_{+}$and $\mathfrak{z}_{+}$, respectively, and let $Y_{j_{-}}=J Y_{j+}, Z_{k-}=J Z_{k+}$. Then, as $\operatorname{ad}_{G_{0}} \mathfrak{u}_{0}=0\left(\left[\mathrm{~A}\right.\right.$, Lemma 4.6]) and $\operatorname{ad}_{H_{0}} \mathfrak{u}_{0}=0$ ([A, (5.2)]), we have the Lie brackets on $\mathfrak{u}$ given in Table 1 .

Furthermore, the Kählerian Lie algebra $(\mathfrak{u}, J)$ has a unique Q-representation on the Euclidean vector space $\tilde{\mathfrak{u}}=\tilde{\mathfrak{f}}_{0}+\widetilde{\mathfrak{u}}_{0}, T: \mathfrak{u} \rightarrow \operatorname{End}(\tilde{\mathfrak{u}})$, where $\sim: \mathfrak{u} \rightarrow \tilde{\mathfrak{u}}$ denotes the corresponding isometry of Euclidean vector spaces.

Consider the quaternion-Hermitian vector space $(\mathfrak{w}(p, q),\langle\rangle, q$,$) , where$ the space $\mathfrak{w}(p, q)=\mathfrak{u}+\tilde{\mathfrak{u}}$ is a direct orthogonal sum, and $q=\operatorname{Span}\left\{J_{a}\right.$ : $a=1,2,3\}$ is the quaternionic structure on $\mathfrak{w}(p, q)$ defined by $(2.7)$. Then

$$
\mathcal{B}=\left\{G_{i}, H_{i}, Y_{j+}, Y_{j-}, Z_{k+}, Z_{k-}, \widetilde{G}_{i}, \widetilde{H}_{i}, \widetilde{Y}_{j+}, \widetilde{Y}_{j_{-}}, \widetilde{Z}_{k+}, \widetilde{Z}_{k-}\right\},
$$


TABLE 1. Lie brackets on $\mathfrak{u}$.

\begin{tabular}{|c||c|c|c|c|c|c|c|c|c|c|c|c|}
\hline$\llbracket \downarrow, \rightarrow]$ & $G_{0}$ & $H_{0}$ & $G_{1}$ & $H_{1}$ & $G_{2}$ & $H_{2}$ & $G_{3}$ & $H_{3}$ & $Y_{j^{\prime}+}$ & $Y_{j^{\prime}-}$ & $Z_{k^{\prime}+}$ & $Z_{k^{\prime}-}$ \\
\hline \hline$G_{0}$ & 0 & $-G_{0}$ & 0 & 0 & 0 & 0 & 0 & 0 & 0 & 0 & 0 & 0 \\
\hline$H_{0}$ & $G_{0}$ & 0 & 0 & 0 & 0 & 0 & 0 & 0 & 0 & 0 & 0 & 0 \\
\hline$G_{1}$ & 0 & 0 & 0 & $-G_{1}$ & 0 & 0 & 0 & 0 & 0 & 0 & 0 & 0 \\
\hline$H_{1}$ & 0 & 0 & $G_{1}$ & 0 & 0 & 0 & 0 & 0 & $\frac{1}{2} Y_{j^{\prime}+}$ & $\frac{1}{2} Y_{j^{\prime}-}$ & $\frac{1}{2} Z_{k^{\prime}+}$ & $\frac{1}{2} Z_{k^{\prime}-}$ \\
\hline$G_{2}$ & 0 & 0 & 0 & 0 & 0 & $-G_{2}$ & 0 & 0 & 0 & 0 & 0 & $Z_{k^{\prime}+}$ \\
\hline$H_{2}$ & 0 & 0 & 0 & 0 & $G_{2}$ & 0 & 0 & 0 & 0 & 0 & $\frac{1}{2} Z_{k^{\prime}+}$ & $-\frac{1}{2} Z_{k^{\prime}-}$ \\
\hline$G_{3}$ & 0 & 0 & 0 & 0 & 0 & 0 & 0 & $-G_{3}$ & 0 & $Y_{j^{\prime}+}$ & 0 & 0 \\
\hline$H_{3}$ & 0 & 0 & 0 & 0 & 0 & 0 & $G_{3}$ & 0 & $\frac{1}{2} Y_{j^{\prime}+}$ & $-\frac{1}{2} Y_{j^{\prime}-}$ & 0 & 0 \\
\hline$Y_{j+}$ & 0 & 0 & 0 & $-\frac{1}{2} Y_{j+}$ & 0 & 0 & 0 & $-\frac{1}{2} Y_{j+}$ & 0 & $\delta_{j j^{\prime}} G_{1}$ & 0 & 0 \\
\hline$Y_{j-}$ & 0 & 0 & 0 & $-\frac{1}{2} Y_{j-}$ & 0 & 0 & $-Y_{j+}$ & $\frac{1}{2} Y_{j-}$ & $-\delta_{j j^{\prime}} G_{1}$ & 0 & 0 & 0 \\
\hline$Z_{k+}$ & 0 & 0 & 0 & $-\frac{1}{2} Z_{k+}$ & 0 & $-\frac{1}{2} Z_{k+}$ & 0 & 0 & 0 & 0 & 0 & $\delta_{k k^{\prime}} G_{1}$ \\
\hline$Z_{k-}$ & 0 & 0 & 0 & $-\frac{1}{2} Z_{k-}-Z_{k+}$ & $\frac{1}{2} Z_{k-}$ & 0 & 0 & 0 & 0 & $-\delta_{k k^{\prime}} G_{1}$ & 0 \\
\hline
\end{tabular}

for $0 \leq i \leq 3,1 \leq j \leq p, 1 \leq k \leq q$, is an orthonormal basis of $\mathfrak{w}(p, q)$.

The action of $J_{a}, a=1,2,3$, on $\mathfrak{w}(p, q)=\mathfrak{u}+\tilde{\mathfrak{u}}$ is given in Table 2. Moreover, the vector space $\mathfrak{w}(p, q)$ has a structure of Lie algebra given

TABLE 2. The action of $J_{a}, a=1,2,3$, on $\mathfrak{w}(p, q)$.

\begin{tabular}{ccccccccccccc}
\hline & $G_{i}$ & $H_{i}$ & $Y_{j+}$ & $Y_{j-}$ & $Z_{k+}$ & $Z_{k-}$ & $\widetilde{G}_{i}$ & $\widetilde{H}_{i}$ & $\widetilde{Y}_{j_{+}}$ & $\widetilde{Y}_{j_{-}}$ & $\widetilde{Z}_{k+}$ & $\widetilde{Z}_{k-}$ \\
\hline$J_{1}$ & $-H_{i}$ & $G_{i}$ & $Y_{j-}$ & $-Y_{j+}$ & $Z_{k-}$ & $-Z_{k+}$ & $\widetilde{H}_{i}$ & $-\widetilde{G}_{i}$ & $-\widetilde{Y}_{j_{-}}$ & $\widetilde{Y}_{j_{+}}$ & $-\widetilde{Z}_{k-}$ & $\widetilde{Z}_{k+}$ \\
$J_{2}$ & $\widetilde{G}_{i}$ & $\widetilde{H}_{i}$ & $\widetilde{Y}_{j_{+}}$ & $\widetilde{Y}_{j}$ & $\widetilde{Z_{k+}}$ & $\widetilde{Z_{k}}$ & $-G_{i}-H_{i}-Y_{j+}$ & $-Y_{j-}$ & $-Z_{k+}$ & $-Z_{k-}$ \\
$J_{3}$ & $\widetilde{H}_{i}$ & $-\widetilde{G}_{i}$ & $-\widetilde{Y}_{j_{-}}$ & $\widetilde{Y}_{j_{+}}$ & $-\widetilde{Z}_{k_{-}}$ & $\widetilde{Z}_{k+}$ & $H_{i}$ & $-G_{i}$ & $-Y_{j-}$ & $Y_{j+}$ & $-Z_{k-}$ & $Z_{k+}$ \\
\hline
\end{tabular}

by (2.8), with $\mathfrak{s}=\mathfrak{w}(p, q)$, where the complex structure $\hat{J}$ on $\tilde{\mathfrak{u}}$ is defined by Table 3 . Hence, by the third condition in (2.8), the nonnull brackets of

TABle 3 . The complex structure $\hat{J}$ on $\tilde{u}$.

\begin{tabular}{rcc}
\hline$\widetilde{G}_{0} \widetilde{H}_{0} \widetilde{G}_{i} \widetilde{H}_{i} \widetilde{Y}_{j_{+}} \widetilde{Y}_{j_{-}}{\widetilde{Z_{k}}}_{\widetilde{Z}_{k-}}$ \\
\hline$\hat{J}-\widetilde{H}_{0} \widetilde{G}_{0} \widetilde{H}_{i}-\widetilde{G}_{i}-\widetilde{Y}_{j_{-}} \widetilde{Y}_{j_{+}}-\widetilde{Z}_{k-} \widetilde{Z}_{k_{+}}$ \\
\hline
\end{tabular}

the elements of $\tilde{\mathfrak{u}}$ are

$$
\left[\widetilde{H}_{0}, \widetilde{G}_{0}\right]=-\left[\widetilde{H}_{i}, \widetilde{G}_{i}\right]=-\left[\widetilde{Y}_{j_{+}}, \widetilde{Y}_{j_{-}}\right]=-\left[\widetilde{Z}_{k_{+}}, \widetilde{Z}_{k_{-}}\right]=G_{0}, \quad i=1,2,3 .
$$

If $U \in \mathfrak{u}$ and $\tilde{V} \in \tilde{\mathfrak{u}}$, then by the second condition in (2.8), one has $[U, \tilde{V}]=$ $T_{U} \tilde{V}$, and the values of $T_{U} \tilde{V}$ are given in Tables 4 and 5 , where $T: \mathfrak{u} \rightarrow$ $\operatorname{End}(\tilde{\mathfrak{u}})$ is expressed in terms of the orthonormal basis $\left\{\widetilde{G}_{i}, \widetilde{H}_{i}, \widetilde{Y}_{j_{+}}, \widetilde{Y}_{j_{-}}\right.$, $\left.\widetilde{Z_{k+}}, \widetilde{Z_{k-}}\right\}$ of $\tilde{\mathfrak{u}}$, from the conditions (Q1-8) of a Q-representation (cf. [Co, 
Prop. 2.1]). Rows 1 to 8 in Table 5 follow from the properties of a weight decomposition with respect to a nondegenerate symplectic representation (2.5) and the properties in [A, Prop. 9.3].

The Lie algebra $\mathfrak{w}(p, q)$ is 4 -step solvable with $\operatorname{dim}_{\mathbb{R}} \mathfrak{w}(p, q)=4(4+p+q)$, and the corresponding simply-connected Lie group with left-invariant metric is the Alekseevskian space $\mathcal{W}(p, q)$.

We have $\mathfrak{w}(p, q)^{*}=\mathfrak{u}^{*}+\tilde{\mathfrak{u}}^{*}$. Let

$$
\mathcal{B}^{*}=\left\{\gamma^{i}, \eta^{i}, \xi^{j+}, \xi^{j-}, \zeta^{k+}, \zeta^{k-}, \tilde{\gamma}^{i}, \tilde{\eta}^{i}, \tilde{\xi}^{j+}, \tilde{\xi}^{j-}, \tilde{\zeta}^{k+}, \tilde{\zeta}^{k-}\right\}
$$

be the basis of $\mathfrak{w}(p, q)^{*}$ dual to the basis (3.1) of $\mathfrak{w}(p, q)$, and denote by $S_{X}$ the 2-form defined by $S_{X}(Y, Z)=S_{X Y Z}$. Then we have the next theorem.

Theorem 3.1. The homogeneous quaternionic Kähler structure $S$ on each rank-four Alekseevskian space $\mathcal{W}(p, q), 0 \leq p \leq q$, which gives its description as the simply-connected solvable Lie group with Lie algebra $\mathfrak{w}(p, q)$, is given, in terms of the basis $\mathcal{B}^{*}$ of $\mathfrak{w}(p, q)^{*}$, by

$$
\begin{aligned}
S_{\mid \mathfrak{u}^{*} \otimes \wedge^{2} \mathfrak{u}^{*}}= & \sum_{i=0}^{3} \gamma^{i} \otimes\left(\gamma^{i} \wedge \eta^{i}\right)-\frac{1}{2}\left(\gamma^{1} \otimes\left(\xi^{j+} \wedge \xi^{j-}+\zeta^{k+} \wedge \zeta^{k-}\right)\right. \\
& \left.+\gamma^{2} \otimes \zeta^{k+} \wedge \zeta^{k-}+\gamma^{3} \otimes \xi^{j+} \wedge \xi^{j-}\right) \\
& +\frac{1}{2} \sum_{j=1}^{p} \xi^{j+} \otimes\left(\xi^{j+} \wedge\left(\eta^{1}+\eta^{3}\right)+\xi^{j-} \wedge\left(\gamma^{1}+\gamma^{3}\right)\right) \\
& +\frac{1}{2} \sum_{j=1}^{p} \xi^{j-} \otimes\left(\xi^{j+} \wedge\left(\gamma^{3}-\gamma^{1}\right)+\xi^{j-} \wedge\left(\eta^{1}-\eta^{3}\right)\right) \\
& +\frac{1}{2} \sum_{k=1}^{q} \zeta^{k+} \otimes\left(\zeta^{k+} \wedge\left(\eta^{1}+\eta^{2}\right)+\zeta^{k-} \wedge\left(\gamma^{1}+\gamma^{2}\right)\right) \\
& +\frac{1}{2} \sum_{k=1}^{q} \zeta^{k-} \otimes\left(\zeta^{k+} \wedge\left(\gamma^{2}-\gamma^{1}\right)+\zeta^{k-} \wedge\left(\eta^{1}-\eta^{2}\right)\right) \\
S_{\mid \mathfrak{u}^{*} \otimes \wedge^{2} \tilde{\mathfrak{u}}^{*}}= & \frac{1}{2} \gamma^{0} \otimes\left(\tilde{\gamma}^{0} \wedge \tilde{\eta}^{0}-\sum_{i=1}^{3} \tilde{\gamma}^{i} \wedge \tilde{\eta}^{i}\right. \\
& \left.+\sum_{j=1}^{p} \tilde{\xi}^{j+} \wedge \tilde{\xi}^{j-}+\sum_{k=1}^{q} \tilde{\zeta}^{k+} \wedge \tilde{\zeta}^{k-}\right) \\
& -\frac{1}{2} \gamma^{1} \otimes\left(\tilde{\gamma}^{0} \wedge \tilde{\eta}^{0}-\tilde{\gamma}^{1} \wedge \tilde{\eta}^{1}+\tilde{\gamma}^{2} \wedge \tilde{\eta}^{2}+\tilde{\gamma}^{3} \wedge \tilde{\eta}^{3}\right) \\
& -\frac{1}{2} \gamma^{2} \otimes\left(\tilde{\gamma}^{0} \wedge \tilde{\eta}^{0}+\tilde{\gamma}^{1} \wedge \tilde{\eta}^{1}-\tilde{\gamma}^{2} \wedge \tilde{\eta}^{2}\right. \\
& \left.+\tilde{\gamma}^{3} \wedge \tilde{\eta}^{3}-\sum_{j=1}^{p} \tilde{\xi}^{j+} \wedge \tilde{\xi}^{j-}\right) \\
& -\frac{1}{2} \gamma^{3} \otimes\left(\tilde{\gamma}^{0} \wedge \tilde{\eta}^{0}+\tilde{\gamma}^{1} \wedge \tilde{\eta}^{1}+\tilde{\gamma}^{2} \wedge \tilde{\eta}^{2}\right. \\
& \left.-\tilde{\gamma}^{3} \wedge \tilde{\eta}^{3}-\sum_{k=1}^{q} \tilde{\zeta}^{k+} \wedge \tilde{\zeta}^{k-}\right) \\
& +\frac{1}{2} \sum_{j=1}^{p} \xi^{j+} \otimes\left(\tilde{\xi}^{j+} \wedge\left(\tilde{\eta}^{1}+\tilde{\eta}^{3}\right)+\tilde{\xi}^{j-} \wedge\left(\tilde{\gamma}^{1}+\tilde{\gamma}^{3}\right)\right) \\
& -\frac{1}{2} \sum_{j=1}^{p} \xi^{j-} \otimes\left(\tilde{\xi}^{j+} \wedge\left(\tilde{\gamma}^{1}-\tilde{\gamma}^{3}\right)-\tilde{\xi}^{j-} \wedge\left(\tilde{\eta}^{1}-\tilde{\eta}^{3}\right)\right) \\
& +\frac{1}{2} \sum_{k=1}^{q} \zeta^{k+} \otimes\left(\tilde{\zeta}^{k+} \wedge\left(\tilde{\eta}^{1}+\tilde{\eta}^{2}\right)+\tilde{\zeta}^{k-} \wedge\left(\tilde{\gamma}^{1}+\tilde{\gamma}^{2}\right)\right) \\
& -\frac{1}{2} \sum_{k=1}^{q} \zeta^{k-} \otimes\left(\tilde{\zeta}^{k+} \wedge\left(\tilde{\gamma}^{1}-\tilde{\gamma}^{2}\right)-\tilde{\zeta}^{k-} \wedge\left(\tilde{\eta}^{1}-\tilde{\eta}^{2}\right)\right), \\
& \frac{1}{2}\left(\sum_{i=0}^{3}\left(\tilde{\gamma}^{i} \wedge \eta^{i}-\tilde{\eta}^{i} \wedge \gamma^{i}\right)\right. \\
& -\sum_{j=1}^{p}\left(\tilde{\xi}^{j+} \wedge \xi^{j-}-\tilde{\xi}^{j-} \wedge \xi^{j+}\right) \\
&
\end{aligned}
$$




$$
\begin{aligned}
& \left.-\sum_{k=1}^{q}\left(\tilde{\zeta}^{k+} \wedge \zeta^{k-}-\tilde{\zeta}^{k-} \wedge \zeta^{k-}\right)\right), \\
& S_{\widetilde{H}_{0}}=\frac{1}{2}\left(\sum_{i=0}^{3}\left(\tilde{\gamma}^{i} \wedge \gamma^{i}+\tilde{\eta}^{i} \wedge \eta^{i}\right)\right. \\
& +\sum_{j=1}^{p}\left(\tilde{\xi}^{j+} \wedge \xi^{j+}+\tilde{\xi}^{j-} \wedge \xi^{j-}\right) \\
& \left.+\sum_{k=1}^{q}\left(\tilde{\zeta}^{k+} \wedge \zeta^{k+}+\tilde{\zeta}^{k-} \wedge \zeta^{k-}\right)\right), \\
& S_{\widetilde{G}_{1}}=\frac{1}{2}\left(\tilde{\gamma}^{0} \wedge \eta^{1}+\tilde{\eta}^{0} \wedge \gamma^{1}+\tilde{\gamma}^{1} \wedge \eta^{0}+\tilde{\eta}^{1} \wedge \gamma^{0}\right. \\
& \left.-\tilde{\gamma}^{2} \wedge \eta^{3}-\tilde{\eta}^{2} \wedge \gamma^{3}-\tilde{\gamma}^{3} \wedge \eta^{2}-\tilde{\eta}^{3} \wedge \gamma^{2}\right), \\
& S_{\widetilde{H}_{1}}=-\frac{1}{2}\left(\tilde{\gamma}^{0} \wedge \gamma^{1}-\tilde{\eta}^{0} \wedge \eta^{1}+\tilde{\gamma}^{1} \wedge \gamma^{0}-\tilde{\eta}^{1} \wedge \eta^{0}\right. \\
& \left.+\tilde{\gamma}^{2} \wedge \gamma^{3}-\tilde{\eta}^{2} \wedge \eta^{3}+\tilde{\gamma}^{3} \wedge \gamma^{2}-\tilde{\eta}^{3} \wedge \eta^{2}\right), \\
& S_{\widetilde{G}_{2}}=\frac{1}{2}\left(\tilde{\gamma}^{0} \wedge \eta^{2}+\tilde{\eta}^{0} \wedge \gamma^{2}-\tilde{\gamma}^{1} \wedge \eta^{3}-\tilde{\eta}^{1} \wedge \gamma^{3}\right. \\
& +\tilde{\gamma}^{2} \wedge \eta^{0}+\tilde{\eta}^{2} \wedge \gamma^{0}-\tilde{\gamma}^{3} \wedge \eta^{1}-\tilde{\eta}^{3} \wedge \gamma^{1} \\
& \left.-\sum_{j=1}^{p}\left(\tilde{\xi}^{j+} \wedge \xi^{j-}+\tilde{\xi}^{j-} \wedge \xi^{j+}\right)\right) \text {, } \\
& S_{\widetilde{H}_{2}}=-\frac{1}{2}\left(\tilde{\gamma}^{0} \wedge \gamma^{2}-\tilde{\eta}^{0} \wedge \eta^{2}+\tilde{\gamma}^{1} \wedge \gamma^{3}-\tilde{\eta}^{1} \wedge \eta^{3}\right. \\
& +\tilde{\gamma}^{2} \wedge \gamma^{0}-\tilde{\eta}^{2} \wedge \eta^{0}+\tilde{\gamma}^{3} \wedge \gamma^{1}-\tilde{\eta}^{3} \wedge \eta^{1} \\
& \left.-\sum_{j=1}^{p}\left(\tilde{\xi}^{j+} \wedge \xi^{j+}-\tilde{\xi}^{j-} \wedge \xi^{j-}\right)\right), \\
& S_{\widetilde{G}_{3}}=\frac{1}{2}\left(\tilde{\gamma}^{0} \wedge \eta^{3}+\tilde{\eta}^{0} \wedge \gamma^{3}-\tilde{\gamma}^{1} \wedge \eta^{2}-\tilde{\eta}^{1} \wedge \gamma^{2}\right. \\
& -\tilde{\gamma}^{2} \wedge \eta^{1}-\tilde{\eta}^{2} \wedge \gamma^{1}+\tilde{\gamma}^{3} \wedge \eta^{0}+\tilde{\eta}^{3} \wedge \gamma^{0} \\
& \left.-\sum_{k=1}^{q}\left(\tilde{\zeta}^{k+} \wedge \zeta^{k-}+\tilde{\zeta}^{k-} \wedge \zeta^{k+}\right)\right), \\
& S_{\widetilde{H}_{3}}=-\frac{1}{2}\left(\tilde{\gamma}^{0} \wedge \gamma^{3}-\tilde{\eta}^{0} \wedge \eta^{3}+\tilde{\gamma}^{1} \wedge \gamma^{2}-\tilde{\eta}^{1} \wedge \eta^{2}\right. \\
& +\tilde{\gamma}^{2} \wedge \gamma^{1}-\tilde{\eta}^{2} \wedge \eta^{1}+\tilde{\gamma}^{3} \wedge \gamma^{0}-\tilde{\eta}^{3} \wedge \eta^{0} \\
& \left.-\sum_{k=1}^{q}\left(\tilde{\zeta}^{k+} \wedge \zeta^{k+}-\tilde{\zeta}^{k-} \wedge \zeta^{k-}\right)\right) \text {, } \\
& S_{\widetilde{Y}_{j_{+}}}=-\frac{1}{2} \sum_{j=1}^{p}\left(\left(\tilde{\gamma}^{0}+\tilde{\gamma}^{2}\right) \wedge \xi^{j-}-\left(\tilde{\eta}^{0}+\tilde{\eta}^{2}\right) \wedge \xi^{j+}\right. \\
& \left.+\tilde{\xi}^{j-} \wedge\left(\gamma^{0}+\gamma^{2}\right)-\tilde{\xi}^{j+} \wedge\left(\eta^{0}+\eta^{2}\right)\right), \\
& S_{\widetilde{Y}_{j_{-}}}=\frac{1}{2} \sum_{j=1}^{p}\left(\tilde{\gamma}^{0}-\tilde{\gamma}^{2}\right) \wedge \xi^{j+}+\left(\tilde{\eta}^{0}-\tilde{\eta}^{2}\right) \wedge \xi^{j-} \\
& \left.+\tilde{\xi}^{j+} \wedge\left(\gamma^{0}-\gamma^{2}\right)+\tilde{\xi}^{j-} \wedge\left(\eta^{0}-\eta^{2}\right)\right), \\
& S_{\widetilde{Z}_{k_{+}}}=-\frac{1}{2} \sum_{k=1}^{q}\left(\left(\tilde{\gamma}^{0}+\tilde{\gamma}^{3}\right) \wedge \zeta^{k-}-\left(\tilde{\eta}^{0}+\tilde{\eta}^{3}\right) \wedge \zeta^{k+}\right. \\
& \left.+\tilde{\zeta}^{k-} \wedge\left(\gamma^{0}+\gamma^{3}\right)-\tilde{\zeta}^{k+} \wedge\left(\eta^{0}+\eta^{3}\right)\right), \\
& S_{\widetilde{Z}_{k_{-}}}=\frac{1}{2} \sum_{k=1}^{q}\left(\left(\tilde{\gamma}^{0}-\tilde{\gamma}^{3}\right) \wedge \zeta^{k+}+\left(\tilde{\eta}^{0}-\tilde{\eta}^{3}\right) \wedge \zeta^{k-}\right. \\
& \left.+\tilde{\zeta}^{k+} \wedge\left(\gamma^{0}-\gamma^{3}\right)+\tilde{\zeta}^{k-} \wedge\left(\eta^{0}-\eta^{3}\right)\right) .
\end{aligned}
$$

Proof. Consider the tensor field $S$ on $\mathcal{W}(p, q), 0 \leq p \leq q$, given by

$$
2\left\langle S_{X} Y, Z\right\rangle=\langle[X, Y], Z\rangle-\langle[X, Z], Y\rangle-\langle[Y, Z], X\rangle,
$$


for $X, Y, Z \in \mathfrak{w}(p, q)$. Let $\nabla$ be the Levi-Civita connection on $\mathcal{W}(p, q)$ with

respect to the invariant metric defined by $\langle$,$\rangle . Then \widetilde{\nabla}=\nabla-S$ is the connection on the Lie group $\mathcal{W}(p, q)$ for which every left-invariant vector field is parallel. Thus, conditions (2.2) are satisfied and $S$ is a homogeneous quaternionic Kähler structure. Moreover, the holonomy algebra of the connection $\widetilde{\nabla}$ is trivial, and then $S$ provides the description of $\mathcal{W}(p, q)$ as a Lie group (see [TV, p. 32, Eqs. (1.79)]).

Since $($ see $(2.8))$ we have $[\mathfrak{u}, \mathfrak{u}] \subset \mathfrak{u},[\mathfrak{u}, \tilde{\mathfrak{u}}] \subset \tilde{\mathfrak{u}},[\tilde{\mathfrak{u}}, \tilde{\mathfrak{u}}] \subset \mathfrak{u}$, and $\mathfrak{u}$ and $\tilde{\mathfrak{u}}$ are orthogonal, from (3.3) we have

$$
S_{U V \widetilde{W}}=0, \quad S_{\widetilde{U} V W}=0, \quad S_{U \widetilde{V} W}=0, \quad S_{\widetilde{U} \widetilde{V} \widetilde{W}}=0 .
$$

On account of (3.3), Table 1 , and equation $S_{U V \widetilde{W}}=0$ in (3.4), one obtains the nonzero values of $S_{U V W}$ for $U, V$ and $W$ in the orthonormal basis $\mathcal{B}$. In order to obtain $S_{\mid \mathfrak{u}^{*} \otimes \bigwedge^{2} \tilde{u}^{*}}$, we use (3.2), (3.3), equation $S_{U \widetilde{V} W}=0$ in (3.4) and Tables 4 and 5 , since $[U, \widetilde{V}]=T_{U} \widetilde{V}$. From (3.3), by using (3.2), Tables 4 and 5, and the equations $S_{\widetilde{U} V W}=S_{\widetilde{U} \widetilde{V} \widetilde{W}}=0$ in (3.4), we obtain the values of $S_{\widetilde{U}}$ for each $\widetilde{U}=\widetilde{G}_{i}, \widetilde{H}_{i}, \widetilde{Y}_{j_{+}}, \widetilde{Y}_{j_{-}}, \widetilde{Z}_{k+}, \widetilde{Z}_{k_{-}}$.

\section{The type of the structure on $\mathcal{W}(p, q)$}

We now determine the type of the previous structure $S$.

Theorem 4.1. The homogeneous quaternionic Kähler structure on each rank-four Alekseevskian space $\mathcal{W}(p, q)$, given in Theorem 3.1, has a nonzero component in each basic Fino's type.

Proof. From the expression of $S$ in Theorem 3.1 and from Table 1 we obtain that the forms $\alpha^{a}, a=1,2,3$, in (2.3) corresponding to $S$ are given by

$$
\alpha^{1}=-\frac{1}{2} \sum_{i=0}^{3} \gamma^{i}, \quad \alpha^{2}=-\tilde{\eta}^{0}, \quad \alpha^{3}=\tilde{\gamma}^{0} .
$$

Hence, since $S=\Theta+\mathcal{T}$, where $\Theta$ is given by (2.3), from (4.1) and using Table 1, it follows that the tensor field $\Theta$ on $\mathcal{W}(p, q)$ corresponding to $S$ is given by

$$
\begin{aligned}
& \frac{1}{4} \sum_{i=0}^{3} \gamma^{i} \otimes\left\{\sum_{l=0}^{3}\left(\gamma^{l} \wedge \eta^{l}-\tilde{\gamma}^{l} \wedge \tilde{\eta}^{l}\right)\right. \\
& \left.\quad-\sum_{j=1}^{p}\left(\xi^{j+} \wedge \xi^{j-}-\tilde{\xi}^{j+} \wedge \tilde{\xi}^{j-}\right)-\sum_{k=1}^{q}\left(\zeta^{k+} \wedge \zeta^{k-}-\tilde{\zeta}^{k+} \wedge \tilde{\zeta}^{k-}\right)\right\} \\
& +\frac{1}{2} \tilde{\gamma}^{0} \otimes\left\{\sum_{l=0}^{3}\left(\gamma^{l} \wedge \tilde{\eta}^{l}+\tilde{\gamma}^{l} \wedge \eta^{l}\right)\right. \\
& \left.2) \quad-\sum_{j=1}^{p}\left(\xi^{j+} \wedge \tilde{\xi}^{j-}+\tilde{\xi}^{j+} \wedge \xi^{j-}\right)-\sum_{k=1}^{q}\left(\zeta^{k+} \wedge \tilde{\zeta}^{k-}+\tilde{\zeta}^{k+} \wedge \zeta^{k-}\right)\right\} \\
& -\frac{1}{2} \tilde{\eta}^{0} \otimes\left\{\sum_{l=0}^{3}\left(\gamma^{l} \wedge \tilde{\gamma}^{l}+\eta^{l} \wedge \tilde{\eta}^{l}\right)\right.
\end{aligned}
$$


HOMOGENEOUS QUATERNIONIC KÄHLER STRUCTURES

TABLE 4. The Q-representation $T: \mathfrak{u} \rightarrow \operatorname{End}(\tilde{\mathfrak{u}})$.

\begin{tabular}{|c|c|c|c|c|}
\hline & $\widetilde{G}_{0}$ & $\widetilde{H}_{0}$ & $\widetilde{G}_{1}$ & $\widetilde{H}_{1}$ \\
\hline$G_{0}$ & 0 & 0 & 0 & 0 \\
\hline$H_{0}$ & $\frac{1}{2} \widetilde{G}_{0}$ & $\frac{1}{2} \widetilde{H}_{0}$ & $\frac{1}{2} \widetilde{G}_{1}$ & $\frac{1}{2} \widetilde{H}_{1}$ \\
\hline$G_{1}$ & $-\frac{1}{2}\left(\widetilde{H}_{0}+\widetilde{H}_{1}\right)$ & $\frac{1}{2}\left(\widetilde{G}_{0}+\widetilde{G}_{1}\right)$ & $\frac{1}{2}\left(\widetilde{H}_{0}+\widetilde{H}_{1}\right)$ & $-\frac{1}{2}\left(\widetilde{G}_{0}+\widetilde{G}_{1}\right)$ \\
\hline$H_{1}$ & $\frac{1}{2} \tilde{G}_{1}$ & $\frac{1}{2} \tilde{H}_{1}$ & $\frac{1}{2} \tilde{G}_{0}$ & $\frac{1}{2} \tilde{H}_{0}$ \\
\hline$G_{2}$ & $-\frac{1}{2}\left(\widetilde{H}_{0}+\widetilde{H}_{2}\right)$ & $\frac{1}{2}\left(\widetilde{G}_{0}+\widetilde{G}_{2}\right)$ & $-\frac{1}{2}\left(\widetilde{H}_{3}+\widetilde{H}_{1}\right)$ & $-\frac{1}{2}\left(\widetilde{G}_{3}-\widetilde{G}_{1}\right)$ \\
\hline $\mathrm{H}_{2}$ & $\frac{1}{2} \tilde{G}_{2}$ & $\frac{1}{2} \tilde{H}_{2}$ & $-\frac{1}{2} \tilde{G}_{3}$ & $\frac{1}{2} \tilde{H}_{3}$ \\
\hline$G_{3}$ & $-\frac{1}{2}\left(\widetilde{H}_{0}+\widetilde{H}_{3}\right)$ & $\frac{1}{2}\left(\widetilde{G}_{0}+\widetilde{G}_{3}\right)$ & $-\frac{1}{2}\left(\widetilde{H}_{1}+\widetilde{H}_{2}\right)$ & $\frac{1}{2}\left(\widetilde{G}_{1}-\widetilde{G}_{2}\right)$ \\
\hline$H_{3}$ & $\frac{1}{2} \tilde{G}_{3}$ & $\frac{1}{2} \tilde{H}_{3}$ & $-\frac{1}{2} \tilde{G}_{2}$ & $\frac{1}{2} \tilde{H}_{2}$ \\
\hline$Y_{j+}$ & $\frac{1}{2} \widetilde{Y}_{j-}$ & $\frac{1}{2} \widetilde{Y}_{j+}$ & $-\frac{1}{2} \widetilde{Y}_{j-}$ & $-\frac{1}{2} \widetilde{Y}_{j+}$ \\
\hline$Y_{j-}$ & $-\frac{1}{2} \widetilde{Y}_{j+}$ & $\frac{1}{2} \widetilde{Y}_{j-}$ & $\frac{1}{2} \widetilde{Y}_{j+}$ & $-\frac{1}{2} \widetilde{Y}_{j-}$ \\
\hline$Z_{k+}$ & $\frac{1}{2} \widetilde{Z}_{k-}$ & $\frac{1}{2} \widetilde{Z}_{k+}$ & $-\frac{1}{2} \widetilde{Z}_{k-}$ & $-\frac{1}{2} \widetilde{Z}_{k+}$ \\
\hline$Z_{k-}$ & $-\frac{1}{2} \widetilde{Z}_{k+}$ & $\frac{1}{2} \widetilde{Z}_{k-}$ & $\frac{1}{2} \widetilde{Z}_{k+}$ & $-\frac{1}{2} \widetilde{Z}_{k-}$ \\
\hline & $\widetilde{G}_{2}$ & $\widetilde{H}_{2}$ & $\widetilde{G}_{3}$ & $\widetilde{H}_{3}$ \\
\hline$G_{0}$ & 0 & 0 & 0 & 0 \\
\hline$H_{0}$ & $\frac{1}{2} \widetilde{G}_{2}$ & $\frac{1}{2} \widetilde{H}_{2}$ & $\frac{1}{2} \widetilde{G}_{3}$ & $\frac{1}{2} \widetilde{H}_{3}$ \\
\hline$G_{1}$ & $-\frac{1}{2}\left(\widetilde{H}_{2}+\widetilde{H}_{3}\right)$ & $\frac{1}{2}\left(\widetilde{G}_{2}-\widetilde{G}_{3}\right)$ & $-\frac{1}{2}\left(\widetilde{H}_{2}+\widetilde{H}_{3}\right)$ & $-\frac{1}{2}\left(\widetilde{G}_{2}-\widetilde{G}_{3}\right)$ \\
\hline$H_{1}$ & $-\frac{1}{2} \tilde{G}_{3}$ & $\frac{1}{2} \tilde{H}_{3}$ & $-\frac{1}{2} \tilde{G}_{2}$ & $\frac{1}{2} \tilde{H}_{2}$ \\
\hline$G_{2}$ & $\frac{1}{2}\left(\widetilde{H}_{0}+\widetilde{H}_{2}\right)$ & $-\frac{1}{2}\left(\widetilde{G}_{0}+\widetilde{G}_{2}\right)$ & $-\frac{1}{2}\left(\widetilde{H}_{3}+\widetilde{H}_{1}\right)$ & $\frac{1}{2}\left(\widetilde{G}_{3}-\widetilde{G}_{1}\right)$ \\
\hline$H_{2}$ & $\frac{1}{2} \tilde{G}_{0}$ & $\frac{1}{2} \tilde{H}_{0}$ & $-\frac{1}{2} \tilde{G}_{1}$ & $\frac{1}{2} \tilde{H}_{1}$ \\
\hline$G_{3}$ & $-\frac{1}{2}\left(\widetilde{H}_{1}+\widetilde{H}_{2}\right)$ & $-\frac{1}{2}\left(\widetilde{G}_{1}-\widetilde{G}_{2}\right)$ & $\frac{1}{2}\left(\widetilde{H}_{0}+\widetilde{H}_{3}\right)$ & $-\frac{1}{2}\left(\widetilde{G}_{0}+\widetilde{G}_{3}\right)$ \\
\hline$H_{3}$ & $-\frac{1}{2} \tilde{G}_{1}$ & $\frac{1}{2} \tilde{H}_{1}$ & $\frac{1}{2} \tilde{G}_{0}$ & $\frac{1}{2} \tilde{H}_{0}$ \\
\hline$Y_{j+}$ & $-\frac{1}{2} \widetilde{Y}_{j-}$ & $\frac{1}{2} \widetilde{Y}_{j+}$ & $-\frac{1}{2} \widetilde{Y}_{j-}$ & $-\frac{1}{2} \widetilde{Y}_{j+}$ \\
\hline$Y_{j-}$ & $-\frac{1}{2} \widetilde{Y}_{j+}$ & $-\frac{1}{2} \widetilde{Y}_{j-}$ & $-\frac{1}{2} \widetilde{Y}_{j+}$ & $\frac{1}{2} \widetilde{Y}_{j-}$ \\
\hline$Z_{k+}$ & $-\frac{1}{2} \widetilde{Z}_{k-}$ & $-\frac{1}{2} \widetilde{Z}_{k+}$ & $-\frac{1}{2} \widetilde{Z}_{k-}$ & $\frac{1}{2} \widetilde{Z}_{k+}$ \\
\hline$Z_{k-}$ & $-\frac{1}{2} \widetilde{Z}_{k+}$ & $\frac{1}{2} \widetilde{Z}_{k-}$ & $-\frac{1}{2} \widetilde{Z}_{k+}$ & $-\frac{1}{2} \widetilde{Z}_{k-}$ \\
\hline
\end{tabular}

$$
\left.+\sum_{j=1}^{p}\left(\xi^{j+} \wedge \tilde{\xi}^{j+}+\xi^{j-} \wedge \tilde{\xi}^{j-}\right)+\sum_{k=1}^{q}\left(\zeta^{k+} \wedge \tilde{\zeta}^{k+}+\zeta^{k-} \wedge \tilde{\zeta}^{k-}\right)\right\}
$$

On the other hand, considering again that the structure decomposes as $S=\Theta+\mathcal{T}$, and the values of the 1 -forms $\alpha^{a}$ are those in (4.1), we have that as, for instance,

$$
\sum_{a=1}^{3} \alpha^{a}\left(J_{a} H_{2}\right)=-\frac{1}{2} \neq 0
$$

the component $\Theta$ of the structure $S$ does not belong to $\mathcal{Q} \mathcal{K}_{2}$. 
TABLE 5. The Q-representation $T: \mathfrak{u} \rightarrow \operatorname{End}(\tilde{\mathfrak{u}})$.

\begin{tabular}{|c|c|c|c|c|}
\hline & $\widetilde{Y}_{j^{\prime}+}$ & $\widetilde{Y}_{j^{\prime \prime}-}$ & $\widetilde{Z}_{k^{\prime}+}$ & $\widetilde{Z}_{k^{\prime}-}$ \\
\hline$\overline{G_{0}}$ & 0 & 0 & 0 & 0 \\
\hline$H_{0}$ & $\frac{1}{2} \widetilde{Y}_{j^{\prime}+}$ & $\frac{1}{2} \widetilde{Y}_{j^{\prime}-}$ & $\frac{1}{2} \widetilde{Z}_{k^{\prime}+}$ & $\frac{1}{2} \widetilde{Z}_{k^{\prime}-}$ \\
\hline$G_{1}$ & 0 & 0 & 0 & 0 \\
\hline$H_{1}$ & 0 & 0 & 0 & 0 \\
\hline$G_{2}$ & 0 & $-\widetilde{Y}_{j^{\prime}+}$ & 0 & 0 \\
\hline$H_{2}$ & $\frac{1}{2} \widetilde{Y}_{j^{\prime}+}$ & $-\frac{1}{2} \widetilde{Y}_{j^{\prime}-}$ & 0 & 0 \\
\hline$G_{3}$ & 0 & 0 & 0 & $-\widetilde{Z_{k+}}$ \\
\hline \multirow[t]{2}{*}{$H_{3}$} & 0 & 0 & $\frac{1}{2} \widetilde{Z}_{k^{\prime}+}$ & $-\frac{1}{2} \widetilde{Z}_{k^{\prime}-}$ \\
\hline & $\widetilde{Y}_{j^{\prime}+}$ & $\widetilde{Y}_{j^{\prime}-}$ & $\widetilde{Z}_{k^{\prime}+}$ & $\widetilde{Z}_{k^{\prime}-}$ \\
\hline$Y_{j+}$ & $\begin{array}{l}\frac{\delta_{j j^{\prime}}}{2}\left(\widetilde{H}_{0}+\widetilde{H}_{1}\right. \\
\left.\quad+\widetilde{H}_{2}+\widetilde{H}_{3}\right)\end{array}$ & $\begin{aligned} \frac{\delta_{j j^{\prime}}}{2}\left(\widetilde{G}_{0}+\widetilde{G}_{1}\right. & \left.-\widetilde{G}_{2}+\widetilde{G}_{3}\right)\end{aligned}$ & 0 & 0 \\
\hline$Y_{j-}$ & $\begin{array}{l}-\frac{\delta_{j j^{\prime}}}{2}\left(\widetilde{G}_{0}+\widetilde{G}_{1}\right. \\
\left.+\widetilde{G}_{2}-\widetilde{G}_{3}\right)\end{array}$ & $\begin{array}{l}\frac{\delta_{j j^{\prime}}}{2}\left(\widetilde{H}_{0}+\widetilde{H}_{1}\right. \\
\left.\quad-\widetilde{H}_{2}-\widetilde{H}_{3}\right)\end{array}$ & 0 & 0 \\
\hline$Z_{k+}$ & 0 & 0 & $\begin{array}{r}\frac{\delta_{k k^{\prime}}}{2}\left(\widetilde{H}_{0}+\widetilde{H}_{1}\right. \\
\left.\widetilde{H}_{2}+\widetilde{H}_{3}\right)\end{array}$ & $\begin{array}{l}\frac{\delta_{k k^{\prime}}}{2}\left(\widetilde{G}_{0}+\widetilde{G}_{1}\right. \\
\left.\quad+\widetilde{G}_{2}-\widetilde{G}_{3}\right)\end{array}$ \\
\hline$Z_{k-}$ & 0 & 0 & $\begin{array}{r}-\frac{\delta_{k k^{\prime}}}{2}\left(\widetilde{G}_{0}+\widetilde{G}_{1}\right. \\
\left.-\widetilde{G}_{2}+\widetilde{G}_{3}\right) \\
\end{array}$ & $\begin{array}{l}\frac{\delta_{k k^{\prime}}}{2}\left(\widetilde{H}_{0}+\widetilde{H}_{1}\right. \\
\left.\quad-\widetilde{H}_{2}-\widetilde{H}_{3}\right) \\
\end{array}$ \\
\hline
\end{tabular}

From (4.2), the nonzero values of $\Theta_{X Y Z}$ are those with $X=G_{0}, G_{1}$, $G_{2}, G_{3}, \widetilde{G}_{0}, \widetilde{H}_{0}$. In particular one has the next nonzero values of type $\Theta_{X X Y}$.

$$
\begin{aligned}
\Theta_{G_{0} G_{0} H_{0}}= & \Theta_{G_{1} G_{1} H_{1}}=\Theta_{G_{2} G_{2} H_{2}}=\Theta_{G_{3} G_{3} H_{3}}=\frac{1}{4}, \\
\Theta_{\widetilde{G}_{0} \widetilde{G}_{0} H_{0}} & =\Theta_{\widetilde{H}_{0} \widetilde{H}_{0} H_{0}}=\frac{1}{2} .
\end{aligned}
$$

Suppose then that $\Theta \in \mathcal{Q} \mathcal{K}_{1}$, so there would be a 1 -form $\alpha$ as that in expressions (2.4), and in particular we would have

$$
\frac{1}{4}=\Theta_{G_{0} G_{0} H_{0}}=\alpha\left(H_{0}\right), \quad \frac{1}{2}=\Theta_{\widetilde{G}_{0} \widetilde{G}_{0} H_{0}}=\alpha\left(H_{0}\right),
$$

which is absurd. Whence $\Theta \in \mathcal{Q K}_{12} \backslash\left\{\mathcal{Q K}_{1} \cup \mathcal{Q} \mathcal{K}_{2}\right\}$.

Furthermore, as $\operatorname{dim} \mathcal{W}(p, q)=4(4+p+q)$ and on account of (4.3), the form $\beta$ defining the $\mathcal{Q} \mathcal{K}_{3}$-component (see expressions (2.4)), that is,

$$
\beta=\frac{1}{2+\operatorname{dim} \mathfrak{w}(p, q)} c_{12}(\mathfrak{T})=\frac{1}{18+4(p+q)} c_{12}(\mathfrak{T}),
$$

is given by

$$
\beta=\frac{1}{18+4(p+q)}\left\{\left(\frac{15}{4}+p+q\right) \eta^{0}+\left(\frac{3}{4}+p+q\right) \eta^{1}+\frac{3}{4}\left(\eta^{2}+\eta^{3}\right)\right\}
$$

Hence $S$ has a nonzero component in $\mathcal{Q} \mathcal{K}_{3}$ for all $0 \leq p \leq q$. 
Consider now the operator $\Psi: \widehat{\mathcal{V}} \rightarrow \widehat{\mathcal{V}}$ defined by

$$
\Psi(\mathcal{T})_{X Y Z}=\mathcal{T}_{Y Z X}+\mathcal{T}_{Z X Y}+\sum_{a=1}^{3}\left(\mathcal{T}_{J_{a} Y J_{a} Z X}+\mathcal{T}_{J_{a} Z X J_{a} Y}\right)
$$

having eigenvalues 2 and -4 , with corresponding eigenspaces $\mathcal{Q K}_{34}$ and $\mathcal{Q} \mathcal{K}_{5}$, respectively (see expressions (2.4)). Consider $\mathcal{T}^{\beta} \in \mathcal{Q} \mathcal{K}_{3}$, given by

$$
\begin{aligned}
\mathcal{T}_{X Y Z}^{\beta}=\langle X, Y\rangle \beta(Z)-\langle X, Z\rangle \beta(Y) & \\
& +\sum_{a=1}^{3}\left(\left\langle X, J_{a} Y\right\rangle \beta\left(J_{a} Z\right)-\left\langle X, J_{a} Z\right\rangle \beta\left(J_{a} Y\right)\right),
\end{aligned}
$$

where $\beta$ stands for the 1 -form (4.4). Then $\mathcal{T}-\mathcal{T}^{\beta} \in \mathcal{Q} \mathcal{K}_{45}$, so that $\Psi(\mathcal{T}-$ $\left.\mathcal{T}^{\beta}\right)_{X Y Z}=\Psi(\mathcal{T})_{X Y Z}-2 \mathcal{T}_{X Y Z}^{\beta}$. Taking then for instance the vectors $X=$ $Y=G_{0}, Z=H_{0}$, we get

$$
\left(\mathcal{T}-\mathcal{T}^{\beta}\right)_{G_{0} G_{0} H_{0}}=\frac{6+p+q}{2(9+2(p+q))}, \quad \Psi\left(\mathcal{T}-\mathcal{T}^{\beta}\right)_{G_{0} G_{0} H_{0}}=-\frac{21+5(p+q)}{9+2(p+q)},
$$

hence $\mathcal{T}-\mathcal{T}^{\beta} \in \mathcal{Q} \mathcal{K}_{45} \backslash\left\{\mathcal{Q} \mathcal{K}_{4} \cup \mathcal{Q} \mathcal{K}_{5}\right\}$ for all $0 \leq p \leq q$. That is, $S$ has, for all $0 \leq p \leq q$, a nonzero component in each basic type.

As the simplest examples, consider the $4(4+q)$-dimensional spaces $\mathcal{W}(0, q) \cong$ $\mathrm{SO}_{0}(4+q, 4) /(\mathrm{SO}(4+q) \times \mathrm{SO}(4)), q \geq 0$, which (cf. [Co, Table 1]) are the Alekseevskian $\mathcal{W}$-spaces which are symmetric. As such, they admit the structure $S=0$. Moreover, as solvable Lie groups with Lie algebra $\mathfrak{w}(0, q)$ they admit the corresponding structure given by Theorem 3.1, when $p=0$.

Acknowledgements. This research was partly supported by MICINN, Spain, Project MTM2008-01386.

\section{REFERENCES}

[A] D. V. Alekseevsky, Classification of quaternionic spaces with a transitive solvable group of motions, Math. USSR Izvestija 9 (1975), 297-339.

[AC] D. V. Alekseevsky and V. Cortés, Isometry groups of homogeneous quaternionic Kähler manifolds, J. Geom. Anal. 9 (1999), 513-545.

[AS] W. Ambrose and I. M. Singer, On homogeneous Riemannian manifolds, Duke Math. J. 25 (1958), 647-669.

[BW] J. Bagger and E. Witten, Matter couplings in $N=2$ supergravity, Nucl. Phys. B222 (1983), 1-10.

[BGO1] W. Batat, P. M. Gadea and J. A. Oubiña, Homogeneous pseudoRiemannian structures of linear type, J. Geom. Phys. 61 (2011), $745-764$. 
[BGO2] W. Batat, P. M. Gadea and J. A. Oubiña, Homogeneous quaternionic Kähler structures on rank-three Alekseevsky spaces, Publ. Math. Debrecen 78 (2011), to appear.

[CGO1] M. Castrillón López, P. M. Gadea and J. A. Oubiña, Homogeneous quaternionic Kähler structures on eight-dimensional non-compact quaternion-Kähler symmetric spaces, Math. Phys. Anal. Geom. 12 (2009), 47-74.

[CGO2] M. Castrillón López, P. M. Gadea and J. A. Oubiña, Homogeneous quaternionic Kähler structures on 12-dimensional Alekseevsky spaces, J. Geom. Phys. 57 (2007), 2098-2113.

[CGS] M. Castrillón López, P. M. Gadea and A. F. Swann, Homogeneous quaternionic Kähler structures and quaternionic hyperbolic space, Transform. Groups 11 (2006), 575-608.

[Ce] S. Cecotti, Homogeneous Kähler manifolds and T-algebras in $N=2$ supergravity and superstrings, Comm. Math. Phys. 124 (1989), 2355.

[Co] V. Cortés, Alekseevskian spaces, Differential Geom. Appl. 6 (1996), $129-168$.

[F] A. Fino, Intrinsic torsion and weak holonomy, Math. J. Toyama Univ. 21 (1998), 1-22.

[I] S. Ishihara, Quaternion Kählerian manifolds, J. Differential Geom. 9 (1974), 483-500.

[J] W. Jelonek, Quaternionic-Kähler geometry and almost Kähler $\mathcal{A}$ manifolds, Ann. Polon. Math. 75 (2000), 111-124.

[K] V. F. Kiričenko, On homogeneous Riemannian spaces with an invariant structure tensor, Dokl. Akad. Nauk SSSR 252 (1980), 291-293.

[TV] F. Tricerri and L. Vanhecke, Homogeneous structures on Riemannian manifolds, London Math. Soc. Lect. Notes Ser. vol. 83, Cambridge Univ. Press, 1983.

[V] G. E. Vîlcu, 3-submersions from QR-hypersurfaces of quaternionic Kähler manifolds, Ann. Polon. Math. 98 (2010), 301-309.

[WP] B. de Wit and A. van Proeyen, Special geometry, cubic polynomials and homogeneous quaternionic spaces, Comm. Math. Phys. 149 (1992), 307-333.

[W] J. A. Wolf, emphComplex homogeneous contact manifolds and quaternionic symmetric spaces, J. Math. Mech. 14 (1965), 10331047. 
(Corresponding author) École Normale Supérieure D’Enseignement Technologique D'Oran, Département de Mathématiques et Informatique, B.P. 1523, El M'NaOuar, Oran, Algeria

E-mail address: wafa.batat@enset-oran.dz

Instituto de Fisica Fundamental (IFF), CSiC, Serrano 113-Bis, 28006MADRID, SPAIN

E-mail address: pmgadea@iff.csic.es

Instituto de Fisica Aplicada (IFA), CSiC, Serrano 144, 28006-Madrid, SPAIN

E-mail address: jaime@iec.csic.es 